\title{
The F-index and coindex of V-Phenylenic Nanotubes and Nanotorus and their molecular complement graphs
}

\author{
Mohammed S. Y. Alsharafi ${ }^{1}$, Abdu Q. Alameri ${ }^{2}$ \\ ${ }^{1}$ Graduate School of Natural and Applied Sciences, Yildiz Technical University, Istanbul, Turkey \\ ${ }^{2}$ Department of Biomedical Engineering, University of Science and Technology, Sana'a, Yemen \\ alsharafi205010@gmail.com, a.alameri2222@gmail.com
}

DOI 10.17586/2220-8054-2021-12-3-263-270

\begin{abstract}
The forgotten topological index was defined to be used in the analysis of chemical structures which often appear in drug molecular graphs. In this paper, we studied the F-index and F-coindex for certain important physico chemical structures such as V-Phenylenic Nanotube $V P H X[m, n]$ and V-Phenylenic Nanotorus $V P H Y[m, n]$ and their molecular complement graph. Moreover, we computed F-polynomial of the V-Phenylenic Nanotubes and Nanotorus. These explicit formulae can correlate the chemical structure of molecular graphs of Nanotubes and Nanotorus to information about their physicochemical structure.
\end{abstract}

Keywords: F-index, F-coindex, V-Phenylenic Nanotubes and Nanotori, molecular graph, molecular complement graph.

Received: 10 April 2021

Revised: 18 April 2021

\section{Introduction}

Chemical graph theory is a part of mathematical chemistry that uses graph theory for mathematically modeling chemical phenomena. Chemical graphs are models of molecules in which atoms are represented by vertices and chemical bonds by edges of a graph. The topological indices explain chemical compound structures and help to predict certain physicochemical properties such as entropy, boiling point, acentric factor, vaporization enthalpy, etc [1]. We denote the V-Phenylenic nanotubes and nanotorus by $\operatorname{VPHX}[m, n]$, and $V P H Y[m, n]$ respectively, where $m$ and $n$ are the number of atoms in rows and columns. Many well-known topological indices of the V-Phenylenic nanotubes and nanotorus have been computed. The forgotten index one of the most important topological indices which preserve the symmetry of molecular structures and provide a mathematical formulation to predict their physical and chemical properties [2]. In this article, in view of structure analysis and mathematical derivation, we find the F-index and coindex of certain molecular graphs nanotubes and nanotorus that are interesting molecular graphs and nano-structures. Since the forgotten topological index and coindex are considered among the most effective topological indices in analysis the QSPR/QSAR with high accuracy, so we intend to compute the F-index and F-coindex for some nanostructures such as V-Phenylenic Nanotube $V P H X[m, n]$ and V-Phenylenic Nanotorus $V P H Y[m, n]$ and their polynomials which are useful for description of some characteristics of nanostructures. Topological indices are the molecular descriptors that describe the structures of chemical compounds and they help us to predict certain physicochemical properties [3]. The first and second Zagreb indices can be regarded as one of the oldest graph invariants which was defined in 1972 by Gutman and Trinajsti [4,5]. The first and second Zagreb indices defined for a molecular graph $G$ as:

$$
M_{1}(G)=\sum_{u v \in E(G)}\left[\delta_{G}(u)+\delta_{G}(v)\right], \quad M_{2}(G)=\sum_{u v \in E(G)} \delta_{G}(u) \delta_{G}(v) .
$$

The first and second Zagreb coindices have been introduced by A. R. Ashrafi, T. Doslic, and A. Hamzeh in 2010 [6]. They are respectively defined as:

$$
\bar{M}_{1}(G)=\sum_{u v \notin E(G)}\left[\delta_{G}(u)+\delta_{G}(v)\right], \quad \bar{M}_{2}(G)=\sum_{u v \notin E(G)} \delta_{G}(u) \delta_{G}(v) .
$$

Furtula and Gutman in 2015 introduced forgotten index (F-index) [7] which defined as:

$$
F(G)=\sum_{v \in V(G)} \delta_{G}^{3}(v)=\sum_{u v \in E(G)}\left(\delta_{G}^{2}(u)+\delta_{G}^{2}(v)\right)
$$

N. De, S. M. A. Nayeem and A. Pal. in 2016 defined forgotten coindex (F-coindex) [8], which defined as:

$$
\bar{F}(G)=\sum_{v \notin V(G)} \delta_{G}^{3}(v)=\sum_{u v \notin E(G)}\left(\delta_{G}^{2}(u)+\delta_{G}^{2}(v)\right) .
$$


Then, Farahani et al. [9-11] computed the first and second Zagreb, first and second Hyper-Zagreb and multiplicative and Redefined Zagreb indices of V-Phenylenic Nanotube $V P H X[m, n]$ and V-Phenylenic Nanotorus $V P H Y[m, n]$ and their polynomials. Ashrafi et al. [12] studied the computing Sadhana polynomial of V-phenylenic nanotubes and nanotori. Alamian et al. [13] studied PI Polynomial of V-Phenylenic Nanotubes and Nanotori, Z. Ahmad et al. [14] presented new results on eccentric connectivity indices of V-Phenylenic nanotube, and there are a lot of researchers who have studied some topological indices on V-Phenylenic Nanotube $\operatorname{VPHX}[m, n]$ and V-Phenylenic Nanotorus $V P H Y[m, n]$ that cannot be all mentioned here. B. Furtula et al. [7] and De, Nilanjan et al. [8] defined the F-index and F-coindex and studied their of some special graph and graph operation. Nanotubes and Nanotorus play an important role in many applications such as Energy storage, Bioelectronics and Optoelectronics. Because of their unique structural, electrical, optical, and mechanical properties, graphene nanosheets drew dramatic attention of academic and industrial research [15] and as nanotubes introduced into graphene could be extremely useful and exploited to generate novel, innovative, and useful materials and devices. Here, we present the F-index and F-coindex and their topological polynomials of V-Phenylenic Nanotube $\operatorname{VPHX}[m, n]$ and V-Phenylenic Nanotorus $V P H Y[m, n]$ which are useful for surveying structure of nanotubes and nanotorus. Any unexplained terminology is standard, typically as in $[16-21]$.

\section{Preliminaries}

In this section, we give some basic and preliminary concepts which we shall use later.

Proposition 2.1 [2,8] Let $G$ be a simple graph on $n$ vertices and $m$ edges. Then:

$$
\begin{aligned}
& F(\bar{G})=n(n-1)^{3}-6 m(n-1)^{2}+3(n-1) M_{1}(G)-F(G), \\
& \bar{F}(G)=(n-1) M_{1}(G)-F(G), \\
& \bar{F}(\bar{G})=2 m(n-1)^{2}-2(n-1) M_{1}(G)+F(G) .
\end{aligned}
$$

Theorem 2.2 $[9,10]$ The first and second Zagreb and Hyper-Zagreb indices of the V-Phenylenic Nanotubes $V P H X[m, n]$ and V-Phenylenic Nanotorus $V P H Y[m, n](\forall m, n \in \mathbb{N}-\{1\})$ (Fig. 1,2) are given by:

$$
\begin{array}{cl}
M_{1}(V P H X[m, n])=54 m n-10 m, & M_{1}(V P H Y[m, n])=54 m n, \\
M_{2}(V P H X[m, n])=81 m n+3 m, & M_{2}(V P H Y[m, n])=81 m n, \\
H M(V P H X[m, n])=4 m(81 n-20), & H M(V P H Y[m, n])=324 m n, \\
H M_{2}(V P H X[m, n])=9 m(81 n-29), & H M_{2}(V P H Y[m, n])=729 m n .
\end{array}
$$

\section{Main results}

In this section, we compute the forgotten topological index and coindex for certain important chemical structures such as line graphs of the V-Phenylenic Nanotubes $V P H X[m, n]$ and V-Phenylenic Nanotorus $V P H Y[m, n]$ $(\forall m, n \in \mathbb{N}-\{1\})$ and their molecular complement graph. Here, we study also F-polynomial of V-Phenylenic Nanotubes and Nanotorus.

\subsection{F-index and coindex of the V-Phenylenic Nanotubes $V P H X[m, n](\forall m, n \in \mathbb{N}-\{1\})$}

Theorem 3.1.1 The F-index of the V-Phenylenic Nanotubes $\operatorname{VPH} X[m, n](\forall m, n \in \mathbb{N}-\{1\})$ (Fig. 1) is given by:

$$
F(V P H X[m, n])=162 m n-38 m \text {. }
$$

Proof. By definition of the F-index $F(G)=\sum_{u v \in E(G)}\left[\delta_{G}^{2}(u)+\delta_{G}^{2}(v)\right]$, and by replacing each $G$ with $V P H X[m, n]$, which yield:

$$
F(V P H X[m, n])=\sum_{u v \in E(V P H X[m, n]}\left[\delta_{V P H X[m, n]}^{2}(u)+\delta_{V P H X[m, n]}^{2}(v)\right] .
$$

And the partitions of the vertex set and edge set $V(V P H X[m, n]), E(V P H X[m, n])$, of V-Phenylenic nanotube are given in Table 1,2 respectively [9].

The edge set of $V P H X[m, n]$ is divided into two edge partitions based on the sum of degrees of the end vertices as:

$$
\begin{aligned}
& E_{5}(V P H X[m, n])=E_{6}^{*}=\{e=u v \in E(V P H X[m, n]): \delta(u)=2, \delta(v)=3\} \\
& E_{6}(V P H X[m, n])=E_{9}^{*}=\{e=u v \in E(V P H X[m, n]): \delta(u)=3, \delta(v)=3\}
\end{aligned}
$$




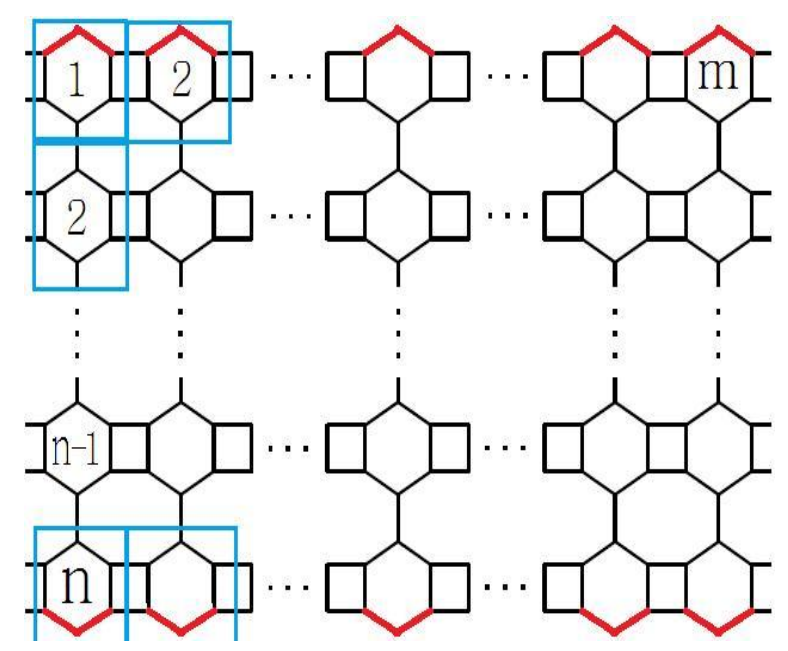

FIG. 1. The molecular graph of $V P H X[m, n]$ nanotube

TABLE 1 . The edge partition of $V P H X[m, n]$ nanotubes

\begin{tabular}{|c|c|c|}
\hline Edge partition & $E_{5}=E_{6}^{*}$ & $E_{6}=E_{9}^{*}$ \\
\hline Cardinality & $4 m$ & $9 m n-5 m$ \\
\hline
\end{tabular}

TABLE 2. The vertex partition of $V P H X[m, n]$ nanotubes

\begin{tabular}{|c|c|c|}
\hline Vertex partition & $V_{2}$ & $V_{3}$ \\
\hline Cardinality & $m+m$ & $6 m n-2 m$ \\
\hline
\end{tabular}

Thus:

$$
\begin{aligned}
F(V P H X[m, n]) & =\sum_{u v \in E(V P H X[m, n])}\left[\delta_{V P H X[m, n]}^{2}(u)+\delta_{V P H X[m, n]}^{2}(v)\right] \\
& =\sum_{u v \in E_{6}^{*}(V P H X[m, n])}\left[\delta_{V P H X[m, n]}^{2}(u)+\delta_{V P H X[m, n]}^{2}(v)\right] \\
& +\sum_{u v \in E_{9}^{*}(V P H X[m, n])}\left[\delta_{V P H X[m, n]}^{2}(u)+\delta_{V P H X[m, n]}^{2}(v)\right] \\
& =13\left|E_{6}^{*}(V P H X[m, n])\right|+18\left|E_{9}^{*}(V P H X[m, n])\right| \\
& =52 m+18[9 m n-5 m] \\
& =162 m n-38 m .
\end{aligned}
$$

Theorem 3.1.2 The F-polynomial of $V P H X[m, n]$ nanotube (Fig. 1) is given by:

$$
F(V P H X[m, n], x)=m\left[4 x^{13}+[9 n-5] x^{18}\right] .
$$

Proof. Since the F-polynomial of graph $G$ is

$$
F(G, x)=\sum_{u v \in E(G)} x^{\left[\delta_{G}^{2}(u)+\delta_{G}^{2}(v)\right]}
$$


And, as Theorem 3.1.1, the partitions of the vertex set and edge set $V(V P H X[m, n]), E(V P H X[m, n])$, of VPhenylenic nanotube are given in Table 1,2 respectively, we have:

$$
\begin{aligned}
F(V P H X[m, n], x) & =\sum_{u v \in E(V P H X[m, n]} x^{\left[\delta_{V P H X[m, n]}^{2}(u)+\delta_{V P H X[m, n]}^{2}(v)\right]} \\
& =\sum_{u v \in E_{6}^{*}(V P H X[m, n])} x^{\left[\delta_{V P H X[m, n]}^{2}(u)+\delta_{V P H X[m, n]}^{2}(v)\right]} \\
& +\sum_{u v \in E_{9}^{*}(V P H X[m, n])} x^{\left[\delta_{V P H X[m, n]}^{2}(u)+\delta_{V P H X[m, n]}^{2}(v)\right]} \\
& =\left|E_{6}^{*}(V P H X[m, n])\right| x^{13}+\left|E_{9}^{*}(V P H X[m, n])\right| x^{18} \\
& =4 m x^{13}+[9 m n-5 m] x^{18} \\
& =m\left[4 x^{13}+[9 n-5] x^{18}\right] .
\end{aligned}
$$

We can also get the F-index of $V P H X[m, n]$ nanotube by derivating the relation F-polynomial of $V P H X[m, n]$ nanotube above as:

$$
\begin{aligned}
F(V P H X[m, n]) & =\left.\frac{\partial Y(V P H X[m, n], x)}{\partial x}\right|_{x=1}=\left.\frac{\partial m\left[4 x^{13}+[9 n-5] x^{18}\right]}{\partial x}\right|_{x=1} \\
& =162 m n-38 m .
\end{aligned}
$$

Corollary 3.1.3 The F-index of complement $V P H X[m, n]$ nanotube (Fig. 1) is given by:

$$
\begin{aligned}
F(\overline{V P H X[m, n]}) & =6 m n[6 m n-1]^{3}-6(9 m n-m)[6 m n-1]^{2} \\
& +3[6 m n-1](54 m n-10 m)-(162 m n-38 m) .
\end{aligned}
$$

Proof. By Proposition 2.1 we have

$$
F(\bar{G})=n(n-1)^{3}-6 m(n-1)^{2}+3(n-1) M_{1}(G)-F(G) .
$$

And $F(V P H X[m, n])=162 m n-38 m$ given in Theorem 3.1.1 above. $M_{1}(V P H X[m, n])=54 m n-10 m$ and the partitions of the vertex set and edge set of $(V P H X[m, n])$ nanotubes are given in [9]:

$$
\sum|V(V P H X[m, n])|=6 m n, \quad \sum|E(V P H X[m, n])|=9 m n-m
$$

Thus:

$$
\begin{aligned}
F(\overline{V P H X[m, n]}) & =\sum|V(V P H X[m, n])|\left[\sum|V(V P H X[m, n])|-1\right]^{3} \\
& -6 \sum|E(V P H X[m, n])|\left[\sum|V(V P H X[m, n])|-1\right]^{2} \\
& +3\left[\sum|V(V P H X[m, n])|-1\right] M_{1}(V P H X[m, n])-F((V P H X[m, n]) \\
& =6 m n[6 m n-1]^{3}-6(9 m n-m)[6 m n-1]^{2} \\
& +3[6 m n-1](54 m n-10 m)-(162 m n-38 m) .
\end{aligned}
$$

Corollary 3.1.4 The F-coindex of $V P H X[m, n]$ nanotube (Fig. 1) is given by:

$$
\bar{F}(V P H X[m, n])=12 m^{2} n(27 n-5)-24 m(9 n-2) \text {. }
$$

Proof. By Proposition 2.1, we have $\bar{F}(G)=(n-1) M_{1}(G)-F(G), F(V P H X[m, n])=162 m n-$ $38 m$ given in Theorem 3.1.1 and $M_{1}(V P H X[m, n])=54 m n-10 m$ given in Theorem 2.2 above and since $n=$ $\sum|V(V P H X[m, n])|=6 m n$. Then:

$$
\begin{aligned}
\bar{F}(V P H X[m, n]) & =\left[\sum|V(V P H X[m, n])|-1\right] M_{1}(V P H X[m, n]) \\
& -F(V P H X[m, n]) \\
& =(6 m n-1)(54 m n-10 m)-(162 m n-38 m) \\
& =12 m^{2} n(27 n-5)-24 m(9 n-2) .
\end{aligned}
$$


TABLE 3. Some topological indices values of $H=V P H X[m, n]$ nanotubes

\begin{tabular}{|c|c|c|c|c|c|c|c|}
\hline$m$ & $n$ & $M_{1}(H)$ & $M_{2}(H)$ & $F(H)$ & $H M(H)$ & $H M_{2}(H)$ & $\bar{F}(H)$ \\
\hline 2 & 2 & 196 & 330 & 572 & 1136 & 2394 & $3.936 \times 10^{3}$ \\
\hline 2 & 3 & 304 & 492 & 896 & 1784 & 3852 & $9.744 \times 10^{3}$ \\
\hline 3 & 2 & 294 & 495 & 858 & 1704 & 3591 & $9.432 \times 10^{3}$ \\
\hline 3 & 3 & 456 & 738 & 1344 & 2676 & 5778 & $22.824 \times 10^{3}$ \\
\hline 4 & 4 & 824 & 1308 & 2440 & 4864 & 10620 & $75.840 \times 10^{3}$ \\
\hline 5 & 5 & 1300 & 2040 & 3860 & 7700 & 16920 & $189.840 \times 10^{3}$ \\
\hline
\end{tabular}

TABLE 4. The edge and vertex partitions of $V P H Y[m, n]$ nanotorus

\begin{tabular}{|c|c|}
\hline Edge partition & $E_{6}=E_{9}^{*}$ \\
\hline Cardinality & $9 m n$ \\
\hline
\end{tabular}

\begin{tabular}{|c|c|}
\hline Vertex partition & $V_{3}$ \\
\hline Cardinality & $6 m n$ \\
\hline
\end{tabular}

Corollary 3.1.5 The F-coindex of complement $V P H X[m, n]$ nanotube (Fig. 1) is given by:

$$
\begin{aligned}
\bar{F}(\overline{V P H X[m, n]}) & =2(9 m n-m)[6 m n-1]^{2}-2[6 m n-1](54 m n-10 m) \\
& +162 m n-38 m
\end{aligned}
$$

Proof. By Proposition 2.1 we have

$$
\bar{F}(\bar{G})=2 m(n-1)^{2}-2(n-1) M_{1}(G)+F(G),
$$

$F(V P H X[m, n])=162 m n-38 m$ given in Theorem 3.1.1 and and $M_{1}(V P H X[m, n])=54 m n-10 m$ given in Theorem 2.2 above and as Corollary 3.1.3 the partitions of the vertex set and edge set of $(V P H X[m, n])$ nanotubes. Then:

$$
\begin{aligned}
\bar{F}(\overline{V P H X[m, n]}) & =2 \sum|E(V P H X[m, n])|\left[\sum|V(V P H X[m, n])|-1\right]^{2} \\
& -2\left[\sum|V(V P H X[m, n])|-1\right] M_{1}(V P H X[m, n])+F(V P H X[m, n]) \\
& =2(9 m n-m)[6 m n-1]^{2}-2[6 m n-1](54 m n-10 m) \\
& +162 m n-38 m .
\end{aligned}
$$

In Table 3 some index and coindex values of $V P H X[m, n]$ nanotubes. formulas reported in Theorem 2.2, Theorem 3.1.1 and Corollary 3.1.4 for the $\operatorname{VPHX}[m, n]$ nanotube. In the table, it shows that values of first and second Zagreb indices, first and second Hyper-Zagreb indices, F-index and F-coindex are in increasing order as the values of $m, n$ increase.

\subsection{F-index and coindex of the V-Phenylenic Nanotorus $V P H Y[m, n](\forall m, n \in \mathbb{N}-\{1\})$}

Theorem 3.2.1 The F-index of the V-Phenylenic Nanotorus $V P H Y[m, n](\forall m, n \in \mathbb{N}-\{1\})$ (Fig. 2) is given by:

$\begin{aligned} & F(V P H Y[m, n])=162 m n . \\ & \text { Proof. By definition of the F-index } F(G)=\sum_{u v \in E(G)}\left[\delta_{G}^{2}(u)+\delta_{G}^{2}(v)\right] \text {, and by replacing each } G \text { with } V P H Y[m, n] \text {, }\end{aligned}$ which yield to $F(V P H Y[m, n])=\sum_{u v \in E(V P H Y[m, n]}\left[\delta_{V P H Y[m, n]}^{2}(u)+\delta_{V P H Y[m, n]}^{2}(v)\right]$, and the partitions of the vertex set and edge set $V(V P H Y[m, n]), E(V P H Y[m, n])$, of V-Phenylenic nanotorus are given in Table 4 respectively [9-11].

The edge set of $V P H Y[m, n]$ have only one type of edges:

$$
E_{6}(V P H Y[m, n])=E_{9}^{*}=\{e=u v \in E(V P H Y[m, n]): \delta(u)=3, \delta(v)=3\} \text {, }
$$




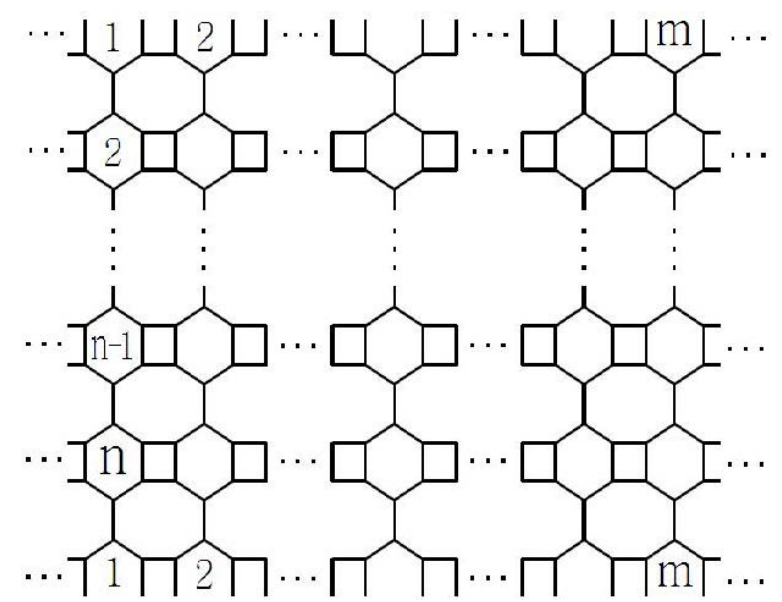

FIG. 2. The molecular graph of $V P H Y[m, n]$ nanotorus

Thus:

$$
\begin{aligned}
F(V P H Y[m, n]) & =\sum_{u v \in E(V P H Y[m, n])}\left[\delta_{V P H Y[m, n]}^{2}(u)+\delta_{V P H Y[m, n]}^{2}(v)\right] \\
& =\sum_{u v \in E_{9}^{*}(V P H Y[m, n])}\left[\delta_{V P H Y[m, n]}^{2}(u)+\delta_{V P H Y[m, n]}^{2}(v)\right] \\
& =18\left|E_{9}^{*}(V P H Y[m, n])\right|=162 m n .
\end{aligned}
$$

Theorem 3.2.2 The F-polynomial of $V P H Y[m, n]$ nanotorus (Fig. 2) is given by:

$$
F(V P H Y[m, n], x)=9 m n x^{18}
$$

Proof. Since the F-polynomial of graph $G$

$$
F(G, x)=\sum_{u v \in E(G)} x^{\left[\delta_{G}^{2}(u)+\delta_{G}^{2}(v)\right]}
$$

And as Theorem 3.2.1 the partitions of the vertex set and edge set $V(V P H Y[m, n]), E(V P H Y[m, n])$, of VPhenylenic nanotorus are given in Table 4 we have:

$$
\begin{aligned}
F(V P H Y[m, n], x) & =\sum_{u v \in E(V P H Y[m, n]} x^{\left[\delta_{V P H Y[m, n]}^{2}(u)+\delta_{V P H Y[m, n]}^{2}(v)\right]} \\
& =\sum_{u v \in E_{9}^{*}(V P H Y[m, n])} x^{\left[\delta_{V P H Y[m, n]}^{2}(u)+\delta_{V P H Y[m, n]}^{2}(v)\right]} \\
& =\left|E_{9}^{*}(\operatorname{VPHY}[m, n])\right| x^{18}=9 m n x^{18} \cdot \square
\end{aligned}
$$

We can also get the F-index of $V P H Y[m, n]$ nanotorus by derivating the relation F-polynomial of $V P H Y[m, n]$ nanotorus above as:

$$
F(V P H Y[m, n])=\left.\frac{\partial F(V P H Y[m, n], x)}{\partial x}\right|_{x=1}=\left.\frac{\partial\left[9 m n x^{18}\right]}{\partial x}\right|_{x=1}=162 m n
$$

Corollary 3.2.3 The F-index of complement $V P H Y[m, n]$ nanotorus (Fig. 2) is given by:

$$
F(\overline{V P H Y[m, n]})=6 m n\left[(6 m n-1)^{3}-9(6 m n-1)^{2}+27(6 m n-2)\right] .
$$

Proof. By Proposition 2.1 we have

$$
F(\bar{G})=n(n-1)^{3}-6 m(n-1)^{2}+3(n-1) M_{1}(G)-F(G),
$$

And $F(V P H Y[m, n])=162 m n$ given in Theorem 3.2.1 above. $M_{1}(V P H Y[m, n])=54 m n$ and the partitions of the vertex set and edge set of $(V P H Y[m, n])$ nanotorus are given in [9].

$$
\sum|V(V P H Y[m, n])|=6 m n, \quad \sum|E(V P H Y[m, n])|=9 m n
$$


TABLE 5. Some topological indices values of $G=V P H Y[m, n]$ nanotorus

\begin{tabular}{|c|c|c|c|c|c|c|c|}
\hline$m$ & $n$ & $M_{1}(G)$ & $M_{2}(G)$ & $F(G)$ & $H M(G)$ & $H M_{2}(G)$ & $\bar{F}(G)$ \\
\hline 2 & 2 & 216 & 324 & 648 & 1296 & 2916 & $4.320 \times 10^{3}$ \\
\hline 2 & 3 & 324 & 486 & 972 & 1944 & 4374 & $10.368 \times 10^{3}$ \\
\hline 3 & 2 & 324 & 486 & 972 & 1944 & 4374 & $10.368 \times 10^{3}$ \\
\hline 3 & 3 & 486 & 729 & 1458 & 2916 & 6561 & $24.300 \times 10^{3}$ \\
\hline 4 & 4 & 864 & 1296 & 2591 & 5184 & 11664 & $79.488 \times 10^{3}$ \\
\hline 5 & 5 & 1350 & 2025 & 4050 & 8100 & 18225 & $197.100 \times 10^{3}$ \\
\hline
\end{tabular}

Thus:

$$
\begin{aligned}
F(\overline{V P H Y[m, n]}) & =\sum|V(V P H Y[m, n])|\left[\sum|V(V P H Y[m, n])|-1\right]^{3} \\
& -6 \sum|E(V P H Y[m, n])|\left[\sum|V(V P H Y[m, n])|-1\right]^{2} \\
& +3\left[\sum|V(V P H Y[m, n])|-1\right] M_{1}(V P H Y[m, n])-F((V P H Y[m, n]) \\
& =6 m n\left[(6 m n-1)^{3}-9(6 m n-1)^{2}+27(6 m n-2)\right] .
\end{aligned}
$$

Corollary 3.2.4 The F-coindex of $V P H Y[m, n]$ nanotorus (Fig. 2) is given by:

$$
\bar{F}(V P H Y[m, n])=54 m n(6 m n-4) .
$$

Proof. By Proposition 2.1 we have $\bar{F}(G)=(n-1) M_{1}(G)-F(G), F(V P H Y[m, n])=162 m n$ given in Theorem 3.1.1 and $M_{1}(V P H Y[m, n])=54 m n$ given in Theorem 2.2 above and since $n=\sum|V(V P H Y[m, n])|=$ $6 m n$. Then:

$$
\begin{aligned}
\bar{F}(V P H Y[m, n]) & =\left[\sum|V(V P H Y[m, n])|-1\right] M_{1}(V P H Y[m, n])-F(V P H Y[m, n]) \\
& =54 m n(6 m n-4) .
\end{aligned}
$$

Corollary 3.2.5 The F-coindex of complement $V P H Y[m, n]$ nanotorus (Fig. 2) is given by:

$$
\bar{F}(\overline{V P H Y[m, n]})=18 m n\left[(6 m n-1)^{2}-36 m n+15\right] .
$$

Proof. By Proposition 2.1 we have

$$
\bar{F}(\bar{G})=2 m(n-1)^{2}-2(n-1) M_{1}(G)+F(G),
$$

$F(V P H Y[m, n])=162 m n$ given in Theorem 3.2.1 and and $M_{1}(V P H X[m, n])=54 m n$ given in Theorem 2.2 above and as Corollary 3.2.3 the partitions of the vertex set and edge set of $(V P H Y[m, n])$ nanotorus. Then:

$$
\begin{aligned}
\bar{F}(\overline{V P H Y[m, n]}) & =2 \sum|E(V P H Y[m, n])|\left[\sum|V(V P H Y[m, n])|-1\right]^{2} \\
& -2\left[\sum|V(V P H Y[m, n])|-1\right] M_{1}(V P H Y[m, n])+F(V P H Y[m, n]) \\
& =18 m n[6 m n-1]^{2}-108 m n[6 m n-1]+162 m n \\
& =18 m n\left[(6 m n-1)^{2}-36 m n+15\right] .
\end{aligned}
$$

In Table 5 some index and coindex values of $V P H Y[m, n]$ nanotorus formulas reported in Theorem 2.2, Theorem 3.1.1 and Corollary 3.2.5 for the $\operatorname{VPHY}[\mathrm{m}, n]$ nanotorus. Table 5 shows that values of first and second Zagreb indices, first and second Hyper-Zagreb indices, F-index and F-coindex are in increasing order as the values of $m, n$ increase. 


\section{Conclusion}

The forgotten index is one of the most important topological indices which preserves the symmetry of molecular structures and provides a mathematical formulation to predict their physical and chemical properties. The present study has computed the F-index and F-coindex of a physico chemical structure of V-Phenylenic Nanotube VPHX[m,n] and V-Phenylenic Nanotorus $\operatorname{VPHY}[m, n]$ and their molecular complement graphs. The study also has computed F-polynomial of V-Phenylenic Nanotube and V-Phenylenic Nanotorus. As the F-index and coindex can been used in QSPR/QSAR study and play a crucial role in analyzing some physico-chemical properties, the results obtained in our paper illustrate the promising prospects of application for nanostructures.

\section{Acknowledgements}

The authors would like to thank the reviewer for his constructive suggestions and useful comments which resulted in an improved version of this paper.

\section{References}

[1] Mondal S., Bhosale A., De N., Pal A. Topological properties of some nanostructures, Nanosystems: Physics, Chemistry, Mathematics, 2020, 11(1),P. 14-24.

[2] Alsharafi M., Shubatah M., Alameri A. The forgotten index of complement graph operations and its applications of molecular graph. Open Journal of Discrete Applied Mathematics, 2020, 3(3), P. 53-61.

[3] Alsharafi M., Shubatah M., Alameri A. The First and Second Zagreb Index of Complement Graph and Its Applications of Molecular Graph. Asian Journal of Probability and Statistics, 2020, 8(3), P. 15-30.

[4] Gutman I., Trinajstic N. Graph theory and molecular orbitals. Total $\pi$-electron energy of alternant hydrocarbons. Chem. Phys. Lett., 1972, 17, P. 535-538.

[5] Khalifeh M., Yousefi-Azari H., Ashrafi A.R. The first and second Zagreb indices of some graph operations. Discrete applied mathematics, 2009, 157(4), P. 804-811.

[6] Ashrafi A., Doslic T., Hamzeh A. The Zagreb coindices of graph operations. Discret. Appl.Math, 2010, 158, P. 1571-1578.

[7] Furtula B., Gutman I. A forgotten topological index. J. Math. Chem., 2015, 53(4), P. 1184-1190.

[8] De., Nilanjan, Sk Md Abu Nayeem, and Anita Pal. The F-coindex of some graph operations. SpringerPlus, 2016, 5(1), P. 221.

[9] Farahani M.R., Kumar R.P., Kanna M.R. First and second zagreb indices and polynomials of V-phenylenic nanotubes and nanotori. Int. J. Pharm. Sci. Res., 2017, 8(1), P. 330-333.

[10] Farahani M.R., Kanna M.R., Kumar R.P. On the Hyper-Zagreb indices of nano-structures. Asian Academic Research Journal of Multidisciplinary, 2016, 3(1), P. 115-123.

[11] Farahani M.R., Wei Gao On Multiplicative and Redefined Version of Zagreb Indices of V-Phenylenic Nanotubes and Nanotorus. BJMCS, 2016, 13(5), P. 1-8.

[12] Ashrafi A.R., Ghorbani M., Jalali M. Computing sadhana polynomial of V-phenylenic nanotubes and nanotori. Ind. J. Chem., 2008, 47A, P. 535-537.

[13] Alamian V., Bahrami A., Edalatzadeh B. PI Polynomial of V-Phenylenic Nanotubes and Nanotori. Int. J. Mol. Sci., 2008, 9, P. $229-234$.

[14] Ahmad Z., Naseem M., Jamil M.K., Siddiquid M.K., Nadeemd M.F. New results on eccentric connectivity indices of V-Phenylenic nanotube. Eurasian Chem. Commun., 2020, 2, P. 663-671.

[15] Furtula B., Gutman I., Dehmer M. On structure-sensitivity of degree-based topological indices. Appl. Math. Comput., 2013, 219, P. $8973-8978$.

[16] Jiang H., Sardar M.S., et al. Computing sanskruti index V-phenylenic nanotube and nanotori. Int. J. Pure Appl. Math., 2017, 115(4), P. 859865.

[17] Alsharafi M., Shubatah M., Alameri A. On the Hyper-Zagreb coindex of some Graphs. J. Math. Comput. Sci., 2020, 10(5), P. $1875-1890$.

[18] Alameri A., Shubatah M., Alsharafi M. Zagreb indices, Hyper Zagreb indices and Redefined Zagreb indices of conical graph. Advances in Mathematics: Scientific Journal, 2020, 9(6), P. 3631-3642.

[19] Kwun Y.C., Munir M., Nazeer W., Rafique S., Kang S.M. M-Polynomials and topological indices of V-Phenylenic Nanotubes and Nanotori. Scientific Reports, 2017, 7(1)P. 8756.

[20] Alsharafi M., Shubatah M. On the Hyper-Zagreb index of some Graph Binary Operations. Asian Research Journal of Mathematics, 2020, 16(4), P. 12-24.

[21] Alsharafi M., Shubatah M., Alameri A. The hyper-Zagreb index of some complement graphs. Advances in Mathematics: Scientific Journal, 2020, 9(6),P. 3631-3642. 